\title{
Increased level of cell-derived microparticles in the cyst fluids of odontogenic keratocysts
}

\author{
QI-WEN MAN ${ }^{1}$, WEN-QUN ZHONG ${ }^{1,2}$, JIAN-GANG REN $^{1,2}$, JIN-YUAN LIU $^{1}$, YUE-YU ZHENG ${ }^{1}$, \\ RUI-FANG LI ${ }^{1,2}$, B. FIDELE NYIMI ${ }^{1}$, GANG CHEN ${ }^{1,2}$, YI-FANG ZHAO ${ }^{1,2}$ and BING LIU ${ }^{1,2}$ \\ ${ }^{1}$ The State Key Laboratory Breeding Base of Basic Science of Stomatology and \\ Key Laboratory of Oral Biomedicine Ministry of Education, School and Hospital of Stomatology, Wuhan University; \\ ${ }^{2}$ Department of Oral and Maxillofacial Surgery, School and Hospital of Stomatology, \\ Wuhan University, Wuhan, Hubei 430079, P.R. China
}

Received October 6, 2017; Accepted March 27, 2018

DOI: $10.3892 /$ ijo.2018.4361

\begin{abstract}
The aim of this study was to examine the level and basic characteristics of cell-derived microparticles (MPs) in the cyst fluids of odontogenic keratocysts (OKCs). For this purpose, MPs from the cyst fluids (CFMPs) of OKCs were purified by a classic differential centrifugation method and characterized by a transmission electron microscope and fluorescence microscope. Flow cytometric analysis was used to determine the size, concentration and cellular origins of the CFMPs. Moreover, the expression level of receptor activator for nuclear factor- $\kappa \mathrm{B}$ ligand in the $\mathrm{OKCs}$ was evaluated by immunohistochemical staining and then analyzed for its correlation with the concentration of CFMPs by Spearman's rank correlation test. In addition, reverse transcription-quantitative polymerase chain reaction (RT-qPCR) and tartaric-resistant acid phosphatase (TRAP) staining were performed to examine the osteoclastogenesis of mouse bone marrow-derived macrophages (BMMs) in response to CFMPs. The results revealed that the levels of total CFMPs were significantly elevated in OKCs compared with dentigerous cysts (DCs) and radicular cysts (RCs). In addition, in vitro experiments further revealed that CFMPs derived from the OKCs of patients could be taken up by BMMs, leading to a significant increase in the mRNA expression levels of nuclear factor of activated T-cells 1 (NFATc1) and TRAP. Moreover, TRAP-positive multinucleated osteoclasts were successfully cultured in the presence of macrophage colony-stimulating factor (M-CSF) and CFMPs with BMMs. On the whole, our findings indicate that patients with OKCs have higher levels of CFMPs compared
\end{abstract}

Correspondence to: Professor Bing Liu, The State Key Laboratory Breeding Base of Basic Science of Stomatology and Key Laboratory of Oral Biomedicine Ministry of Education, School and Hospital of Stomatology, Wuhan University, 237 Luoyu Road, Wuhan, Hubei 430079, P.R. China

E-mail: liubing9909@whu.edu.cn

Key words: cyst fluid, odontogenic keratocysts, microparticles, bone resorption with patients with DCs and RCs, which may be associated with the bone resorption of OKCs.

\section{Introduction}

Odontogenic cysts of the jaws, which are space-occupying lesions, are generally classified into two groups: inflammatory cysts and developmental cysts (1). Inflammatory cysts mainly include radicular cysts (RCs), while the developmental cysts include dentigerous cysts (DCs) and odontogenic keratocysts (OKCs) (2). RCs, which originate from cell rests of Malassez, are the most common jaw inflammatory cysts $(52.2 \%)$ (3). DCs are associated with the crown of an unerupted tooth (4). After being considered as benign tumors for many years (5), OKCs have been moved back into the cyst category by the World Health Organization in 2017, due to their completely regress following decompression and other inadequate evidence defining their neoplastic nature (6).

Cell-derived microparticles (MPs) are small (100-1,000 nm in diameter) membrane-enclosed vesicles secreted from cells by direct budding from the plasma membrane (7). Previous studies by our group have demonstrated that the level of circulating MPs in patients with oral cancer are significantly increased and closely associated with their clinical characteristics, such as blood hypercoagulation and tumor angiogenesis $(8,9)$. Apart from plasma, increased levels of MPs have been also reported in various types of body fluids, such as urine, ascites and pleural effusions in patients with malignant tumors (10-12). MPs highly express phosphatidylserine (PS) on their surface (13), and thus they can be easily detected by fluorescently-labeled Annexin V (14). Additionally, subpopulations of MPs with different cellular origins can be determined by specific markers carried on their membrane surface $(15,16)$.

Since odontogenic cysts are directly immersed in the milieu of cyst fluids, the epithelial cells or surrounding cells of odontogenic cysts may release MPs into the cavity. In the present study, the levels of total and subtype MPs derived from the cyst fluids (CFMPs) were examined and compared among patients with DCs, RCs and OKCs. In addition, the potential clinical significance and biological function of CFMPs were investigated. 


\section{Materials and methods}

Study population. This study was approved by the Review Board of the Medical Ethics Committee of the Hospital of Stomatology, Wuhan University, Wuhan, China and conducted from April, 2016 to February, 2017. This study included a total of 26 patients with OKCs, 20 patients with RCs and 13 patients with DCs (Tables I-III). The diagnosis of DCs, RCs and OKCs was made according to clinical, histopathological (5) and imaging characteristics (17). All patients agreed to participate in the study and signed informed consent forms.

Cyst fluid collection and CFMP isolation. Samples of cystic fluids were obtained from the cyst cavity by aspiration with a syringe attached to an $18 \mathrm{G}$ sterilized needle prior to surgery. The cyst fluids were immediately centrifuged at $3,000 \mathrm{x} \mathrm{g}$ for $20 \mathrm{~min}$ at $4^{\circ} \mathrm{C}$ using the Centrifuge 5810R (Eppendorf, Hamburg, Germany). Supernatants were collected and centrifuged at $3,000 \mathrm{x} \mathrm{g}$ for a further $20 \mathrm{~min}$ to obtain cell-free cyst fluids. Subsequently, an equal volume of $\mathrm{Ca}^{+} / \mathrm{Mg} 2^{+}$-free highly purified phosphate-buffered saline (PBS; Beyotime, Shanghai, China) was added to dilute the supernatants and the dilution was centrifuged at $10,000 \mathrm{x}$ g for $40 \mathrm{~min}$. This step was aimed to remove apoptotic bodies and larger vesicles. The supernatants were then centrifuged at $50,000 \mathrm{x} \mathrm{g}$ for $1 \mathrm{~h}$ at $4^{\circ} \mathrm{C}$ using the Avanti J-26 XP high-speed centrifuge (Beckman Coulter, Irving, TX, USA) to pellet the CFMPs as previously described (8). The CFMP pellets were resuspended with $150 \mu 1$ of PBS. Subsequently, $50 \mu 1$ of the CFMP sample was prepared for flow cytometry and the other $100 \mu \mathrm{l}$ was immediately stored at $-80^{\circ} \mathrm{C}$ for further analyses.

Characterization of CFMPs by transmission electron microscopy (TEM). TEM was performed at the Wuhan Institute of Virology, Chinese Academy of Sciences (Wuhan, China). Freshly-isolated CFMPs from patients with DCs, RCs and OKCs were placed on a copper grid. The grids were stained with $1 \% \mathrm{v} / \mathrm{v}$ uranyl acetate and the samples were examined on a HT7700 transmission electron microscope (Hitachi High-Tech; Hitachi, Tokyo, Japan) as previously described (18).

Characterization of CFMPs by carboxyfluorescein succinimidyl ester (CFSE) labeling. CFSE is a fluorescent dye used for the labeling of MPs (19). It is colorless outside of MPs; however, it can passively diffuse into MPs and become brightly fluorescent after its cleavage of acetate groups by esterases, which are present in MPs (19). For CFSE labeling, the CFMPs were incubated with $10 \mu \mathrm{M}$ CFSE (Sigma-Aldrich, St. Louis, MO, USA) at $37^{\circ} \mathrm{C}$ for $30 \mathrm{~min}$ in the dark. Subsequently, the samples were observed under a fluorescence microscope (Leica Microsystems, Wetzlar, Germany) and analyzed using a BD FACSAria II flow cytometer (BD Biosciences, San Jose, CA, USA) as previously described (8).

Detection and quantification of CFMPs by flow cytometry. Flow cytometric analysis was performed with a BD FACSAria II flow cytometer (BD Biosciences) as described in our previous studies $(8,9)$. The size of the CFMPs was identified with the Nile Red particles with a diameter of 0.7 to $0.9 \mu \mathrm{m}$ (Spherotech, Lake Forest, IL, USA). To quantify the CFMPs, the known number of calibrator flow-count fluorosphere beads (10 $\mu \mathrm{m}$ diameter; Beckman Coulter) were added to determine the number of CFMPs. As flow-count fluorospheres have a definite concentration, when identical volumes of a sample and flow-count fluorospheres are added and tested, the concentration of CFMPs can be calculated using the following formula: (Total number of events for the sample/total number of events for flow-count fluorospheres) $\mathrm{x}$ flow-count fluorospheres assayed concentration. The concentrations of CFMPs were then further calculated by considering the initial volumes of cyst fluids that are listed in Tables I-III. The subpopulations of CFMPs were detected according to the expression of membrane-specific antigens as described in our previous studies $(8,9)$ with minor modifications. The subsets of the CFMPs were identified as follows: Platelet-derived MPs (PMPs, $\mathrm{CD} 31^{+} / \mathrm{CD} 41^{+}$), endothelium-derived MPs (EMPs, CD144 ${ }^{+} /$Annexin $\mathrm{V}^{+}$), erythrocyte-derived MPs (ErMPs, CD235a ${ }^{+} /$Annexin $\mathrm{V}^{+}$),

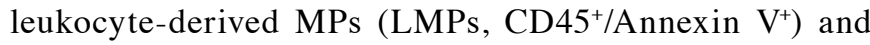
epithelium-derived MPs (EpMPs, EpCAM ${ }^{+} / A n n e x i n \mathrm{~V}^{+}$). The antibodies were added to $10 \mu \mathrm{l}$ of CFMPs as follows: $2 \mu \mathrm{l}$ of anti-CD31-APC-Cy7 (cat. no. 563653; BD Biosciences), anti-CD41-Pacific Blue (cat. no. 305650; BioLegend, Inc., San Diego, CA, USA), anti-CD144-PE-Cy7 (order no. 130-100-722, lot no. 5160926503; Miltenyi Biotec, Bergisch Gladbach, Germany), anti-EpCAM-Qdot605 (cat. no. 563182); $1.5 \mu \mathrm{l}$ of anti-Annexin V-PerCP-Cy5.5 (cat. no. 561431), anti-CD235a-Qdot655 (cat. no. 740583), anti-CD45-Alexa Fluor 700 (cat. no. 560566) (all from BD Biosciences), anti-RANKL-APC (cat. no. FAB6264A; R\&D Systems, Minneapolis, MN, USA) and IgG (as a control) (cat. no. 555749; BD Biosciences). As for incubation buffers, Annexin V Binding Buffer containing calcium (BD Biosciences) was used for anti-Annexin V-PerCP-Cy5.5 detection, whereas PBS was used as a control for the other antibodies. These antibodies and binding buffers were mixed with samples for $30 \mathrm{~min}$ at $4^{\circ} \mathrm{C}$ in the dark. Subsequently, $370 \mu \mathrm{l}$ of PBS was added to the CFMP samples and analyzed by flow cytometry. The results were calculated using FlowJo 9.3.2 software.

Immunohistochemistry. Samples from 24 patients (2 cases were lacking the epithelial component in the IHC test) with OKCs were fixed in $4 \%$ paraformaldehyde and embedded in paraffin under the guidelines of the National Institutes of Health. The tissue samples and CFMPs used for the corollary analyses were collected from the same patient. In brief, the sections were dewaxed, rehydrated and antigen-retrieved by high pressure. Subsequently, the sections were incubated with $3 \%$ hydrogen peroxide for $20 \mathrm{~min}$, goat serum for $20 \mathrm{~min}$ under room temperature, followed by incubation with the primary antibody [receptor activator for nuclear factor- $\kappa \mathrm{B}$ ligand (RANKL), 1:200, Proteintech Group, Wuhan, China; 23408-1-AP] at $4^{\circ} \mathrm{C}$ overnight. For the staining, a diaminobenzidine substrate kit, and hematoxylin (both from Dako, Glostrup, Denmark) were used. For RANKL evaluation, the semi-quantitative analysis of immunohistochemical staining was performed using Image-Pro Plus 6.0 and the quantification was calculated as the mean density for each protein (IOD/area).

DNA electrophoresis. Total RNA was isolated from the CFMPs and the supernatants of OKCs using TRIzol reagent (Invitrogen, 
Table I. Summary of clinical characteristics of patients with odontogenic keratocysts (OKCs).

\begin{tabular}{|c|c|c|c|c|}
\hline $\begin{array}{l}\text { Patient } \\
\text { No. }\end{array}$ & Sex & $\begin{array}{c}\text { Age } \\
\text { (years) }\end{array}$ & Location & $\begin{array}{c}\text { Cyst fluid } \\
\text { drained }(\mathrm{ml})\end{array}$ \\
\hline 1 & $\mathrm{~F}$ & 35 & Left mandible & 1 \\
\hline 2 & $\mathrm{~F}$ & 26 & Right maxilla & 3 \\
\hline 3 & $\mathrm{~F}$ & 39 & Mandible & 4.5 \\
\hline 4 & $\mathrm{~F}$ & 47 & Left mandible & 6 \\
\hline 5 & $\mathrm{~F}$ & 24 & Left mandible & 2.5 \\
\hline 6 & $\mathrm{~F}$ & 24 & Right mandible & 2 \\
\hline 7 & M & 32 & Left mandible & 4.5 \\
\hline 8 & M & 52 & Left mandible & 1 \\
\hline 9 & M & 79 & Right mandible & 5 \\
\hline 10 & M & 24 & Right mandible & 2 \\
\hline 11 & $\mathrm{~F}$ & 11 & Right mandible & 5 \\
\hline 12 & $\mathrm{~F}$ & 52 & Right maxilla & 2 \\
\hline 13 & $\mathrm{~F}$ & 45 & Right mandible & 3 \\
\hline 14 & $\mathrm{M}$ & 34 & Left mandible & 0.8 \\
\hline 15 & $\mathrm{~F}$ & 28 & Right mandible & 3 \\
\hline 16 & $\mathrm{M}$ & 37 & Left maxilla & 7 \\
\hline 17 & M & 26 & Right maxilla & 4 \\
\hline 18 & $\mathrm{M}$ & 24 & Left mandible & 1 \\
\hline 19 & $\mathrm{~F}$ & 32 & Left mandible & 1 \\
\hline 20 & M & 16 & Right mandible & 2 \\
\hline 21 & $\mathrm{~F}$ & 30 & Left maxilla & 2 \\
\hline 22 & $\mathrm{~F}$ & 18 & Right mandible & 1 \\
\hline 23 & $\mathrm{~F}$ & 27 & Maxilla & 1 \\
\hline 24 & $\mathrm{~F}$ & 47 & Right maxilla & 2 \\
\hline 25 & M & 23 & Right mandible & 2 \\
\hline 26 & M & 17 & Left maxilla & 1 \\
\hline
\end{tabular}

M, male; F, female.

Carlsbad, CA, USA) according to the manufacturer's instructions. RNA was reverse transcribed into complementary DNA (cDNA) with Oligo(dT) and AMV reverse transcriptase (Fermentas/Thermo Fisher Scientific, Waltham, MA, USA). The cDNA was then used for polymerase chain reaction (PCR) by amplifying 36 cycles for RANKL $\left(94^{\circ} \mathrm{C}\right.$ for $30 \mathrm{sec}, 60^{\circ} \mathrm{C}$ for $1 \mathrm{~min}, 72^{\circ} \mathrm{C}$ for $1 \mathrm{~min}$, and final elongation at $72^{\circ} \mathrm{C}$ for $10 \mathrm{~min}$ ) on a 7900 HT Real-time PCR System (Applied Biosystems, New York, NY, USA). The primer sequences were as follows: RANKL forward, 5'-TCAGAAGATGGCACTCACTG-3' and reverse, 5'-AACATCTCCCACTGGCTGTA-3'. PCR products were separated in $1 \%$ agarose gels and stained with GelRed (Biotium, Fremont, CA, USA). The blots were imaged with a Syngene G-Box (Syngene, Cambridge, UK).

Co-culture of CFMPs with human immortalized oral epithelial cells (HIOECs). HIOECs were kindly provided by Professor San-Gang He from the School of Stomatology, Wuhan University. The HIOECs were plated in 6-well plates and cultured in defined keratinocyte serum-free medium (Gibco, Carlsbad, CA, USA). When the HIOECs were grown
Table II. Summary of clinical characteristics of patients with dentigerous cysts (DCs).

\begin{tabular}{ccclc}
\hline $\begin{array}{l}\text { Patient } \\
\text { No. }\end{array}$ & Sex & $\begin{array}{c}\text { Age } \\
\text { (years) }\end{array}$ & \multicolumn{1}{c}{ Location } & $\begin{array}{c}\text { Cyst fluid } \\
\text { drained (ml) }\end{array}$ \\
\hline 1 & F & 30 & Left maxilla & 5 \\
2 & F & 18 & Right mandible & 2 \\
3 & M & 18 & Maxilla & 3 \\
4 & F & 33 & Right maxilla & 2 \\
5 & M & 51 & Right mandible & 9 \\
6 & M & 42 & Left maxilla & 2 \\
7 & F & 50 & Left mandible & 11 \\
8 & M & 34 & Right mandible & 3.5 \\
9 & M & 34 & Right mandible & 2 \\
10 & M & 56 & Right mandible & 4 \\
11 & F & 28 & Left maxilla & 5 \\
12 & M & 36 & Right maxilla & 4 \\
13 & F & 33 & Left mandible & 5 \\
\hline
\end{tabular}

M, male; F, female.

Table III. Summary of clinical characteristics of patients with radicular cysts (RCs).

\begin{tabular}{|c|c|c|c|c|}
\hline $\begin{array}{l}\text { Patient } \\
\text { No. }\end{array}$ & Sex & $\begin{array}{c}\text { Age } \\
\text { (years) }\end{array}$ & Location & $\begin{array}{c}\text { Cyst fluid } \\
\text { drained (ml) }\end{array}$ \\
\hline 1 & M & 14 & Left mandible & 4 \\
\hline 2 & $\mathrm{~F}$ & 45 & Right maxilla & 0.8 \\
\hline 3 & $\mathrm{~F}$ & 44 & Left mandible & 7.5 \\
\hline 4 & M & 54 & Right maxilla & 10 \\
\hline 5 & $\mathrm{~F}$ & 34 & Maxilla & 2 \\
\hline 6 & $\mathrm{~F}$ & 63 & Right mandible & 4 \\
\hline 7 & $\mathrm{~F}$ & 32 & Right maxilla & 3 \\
\hline 8 & $\mathrm{M}$ & 7 & Left maxilla & 2 \\
\hline 9 & $\mathrm{M}$ & 43 & Left mandible & 4 \\
\hline 10 & $\mathrm{M}$ & 32 & Mandible & 7 \\
\hline 11 & $\mathrm{~F}$ & 44 & Left mandible & 7.5 \\
\hline 12 & $\mathrm{M}$ & 66 & Left maxilla & 3 \\
\hline 13 & M & 29 & Left maxilla & 2 \\
\hline 14 & $\mathrm{~F}$ & 73 & Right maxilla & 5 \\
\hline 15 & $\mathrm{~F}$ & 51 & Right maxilla & 3 \\
\hline 16 & $\mathrm{M}$ & 8 & Right maxilla & 5 \\
\hline 17 & $\mathrm{M}$ & 17 & Left mandible & 3 \\
\hline 18 & $\mathrm{M}$ & 24 & Left mandible & 6 \\
\hline 19 & $\mathbf{M}$ & 65 & Right mandible & 5 \\
\hline 20 & $\mathrm{M}$ & 41 & Right mandible & 4 \\
\hline
\end{tabular}

$\mathrm{M}$, male; F, female.

to $80 \%$ confluence, they were co-cultured with the CFMPs (5 and $10 \mu \mathrm{g} / \mathrm{ml}$; collected from 2 patients with OKCs) for $48 \mathrm{~h}$.

Cellular uptake assay. This study was approved by the Medical Ethics Committee of Hospital of Stomatology, Wuhan 
University. To investigate the biological functions of CFMPs, cellular uptake assays were performed. For this purpose, 10 six-week-old male C57BL/6 mice were obtained from the Experimental Animal Centre of Wuhan University. The weight of these mice ranged from 15.2 to $18.4 \mathrm{~g}$. The mice were injected intraperitoneally with a lethal dose of sodium pentobarbital $(150 \mathrm{mg} / \mathrm{kg}$ ) followed by cervical dislocation. The whole body of the mice were disinfected with $70 \%$ ethanol by soaking all the fur. The skin of the neck region was cut open, and the muscle attachments of the mandible were removed. Subsequently, the mandible was divided into 2 sections and pulled out. Mandibles were kept in $\alpha$-minimal essential medium ( $\alpha$-MEM) supplemented with $10 \%$ fetal bovine serum (FBS) (both from Gibco), $1 \%$ antibiotics $(100 \mathrm{U} / \mathrm{ml}$ penicillin and $100 \mu \mathrm{g} / \mathrm{ml}$ streptomycin; Sigma-Aldrich). The bone marrow was then flushed with PBS, filtered, and spun down at 1,000 rpm for $5 \mathrm{~min}$. The supernatant was discarded and the cells were resuspended in $1 \mathrm{ml}$ complete $\alpha$-MEM medium. Bone marrow cells were collected and cultured for $12 \mathrm{~h}$ in $\alpha$-MEM containing $10 \%$ FBS in Petri dishes. Subsequently, floating cells were collected and plated in 96-well plates at a density of $3 \times 10^{4}$ cells/well, then cultured for 3 days in $\alpha$-MEM supplemented with $10 \%$ FBS and macrophage colony-stimulating factor (M-CSF; $20 \mathrm{ng} / \mathrm{ml}$; R\&D Systems). Adherent cells were regarded as bone marrow-derived macrophages (BMMs). The HIOECs were plated in 6-well plates and cultured in defined keratinocyte serum-free medium (Gibco). The BMMs and HIOECs were labeled with CellMask $(1: 2,000$, Life Technologies, Carlsbad, CA, USA) at $37^{\circ} \mathrm{C}$ for $30 \mathrm{~min}$ in the dark and cultured on coverslips as described in our previous studies $(8,9)$ with some modifications. The CFMPs from the OKCs $(10 \mu \mathrm{g})$ were labeled with CFSE, and then added to the CellMask-labeled BMMs and HIOECs, which were co-cultured for a period of $2 \mathrm{~h}$. The BMMs and HIOECs were then fixed in $4 \%$ paraformaldehyde for $10 \mathrm{~min}$, stained with 4',6-diamidino-2-phenylindole (DAPI) (cat. no. ZLI-9557; Beijing Zhongshan Golden Bridge Biotechnology, Beijing, China) for $15 \mathrm{~min}$ and observed under a fluorescence microscope (Leica Microsystems).

Cell viability assay. Cell proliferation was measured by 3-(4,5-dimethylthiazol-2-yl)-2,5-diphenyltetrazolium bromide (MTT) assay. The HIOECs and BMMs were seeded in a 96-well culture plate $\left(3 \times 10^{3}\right.$ cells/well) and incubated overnight at $37^{\circ} \mathrm{C}$ with a supply of $5 \% \mathrm{CO}_{2}$. The cells were treated with or without various concentrations of CFMPs (5 or $10 \mu \mathrm{g} / \mathrm{ml})$ and incubated for 24 or $48 \mathrm{~h}$. The cells were washed with PBS, and treated with $20 \mu \mathrm{l}$ of MTT $(5 \mathrm{mg} / \mathrm{ml})$ and incubated for $2 \mathrm{~h}$ at $37^{\circ} \mathrm{C}$ in a $\mathrm{CO}_{2}$ incubator. The blue formazan products formed in the cells were dissolved in $100 \mu 1$ dimethyl sulfoxide (DMSO) and measured at $450 \mathrm{~nm}$ using a spectrophotometer (Power Wave XS2, serial no. 238093; BioTek Instruments, Inc., Winooski, VT, USA). The absorbance values were compared with those of the control cells by GraphPad Prism 6.0 software and shown graphically as the percentage (\%) of viable cells compared to the control.

Western blot analysis. Western blot analysis was performed using the CFMPs and CFMPs co-cultured with the HIOECs. The CFMPs and HIOECs were lysed in RIPA buffer, exposed to brief sonication, and proteins were quantified using the bicinchoninic acid assay (BCA). Subsequently, $40 \mu \mathrm{g}$ of protein were separated by electrophoresis on a $10 \%$ gel SDS-PAGE, and then transferred onto polyvinylidene fluoride (PVDF) membranes. The membranes were incubated in 5\% non-fat milk solution for $1 \mathrm{~h}$ at room temperature followed by overnight incubation at $4{ }^{\circ} \mathrm{C}$ with the following primary antibodies: RANKL (1:2,000, rabbit; 23408-1-AP; Proteintech Group, Wuhan, China) and GAPDH (1:2,000, mouse, \#5174; Cell Signaling Technology, Danvers, MA, USA). The membranes were then incubated for $1 \mathrm{~h}$ at room temperature with an HRP-conjugated secondary antibody (\#7074; Cell Signaling Technology). Following the application of chemiluminescent substrates for the detection of HRP (Life Technologies) at room temperature, the blots were developed with ECL detection reagents (Sigma-Aldrich).

Induction of osteoclast differentiation of BMMs. The adherent BMMs were cultured for 5 days in $\alpha$-MEM supplemented with FBS (10\%), recombinant mouse M-CSF $(20 \mathrm{ng} / \mathrm{ml})$ (R\&D Systems), mouse recombinant sRANKL (50 ng/ml; PeproTech, London, UK), anti-RANKL monoclonal antibody (1,000 ng/ml; R\&D Systems) or CFMPs (5 and $10 \mu \mathrm{g} / \mathrm{ml})$, all of which were replaced every 2 days in 96 -well plates. The cells were fixed with $4 \%$ paraformaldehyde for $10 \mathrm{~min}$ at room temperature and stained for tartaric-resistant acid phosphatase (TRAP) activity. For RNA isolation, the BMMs were seeded on 6-well plates and cultured for 5 days. The BMMs were co-cultured with M-CSF (20 ng/ml) and CFMPs isolated from 2 patients with OKCs at the concentration of 5 or $10 \mu \mathrm{g} / \mathrm{ml}$. Both M-CSF (20 ng/ml)- and sRANKL (50 ng/ml)-treated groups were selected as positive controls.

Reverse transcription-quantitative polymerase chain reaction $(R T-q P C R)$. The isolation of total RNA, synthesis of cDNA and RT-qPCR were performed. Total RNA was extracted from the BMMs using TRIzol reagent (Invitrogen). Subsequently, $2 \mu \mathrm{g}$ of RNA was reverse transcribed into $20 \mu \mathrm{l}$ of cDNA with Oligo(dT) and AMV reverse transcriptase (Fermentas/Thermo Fisher Scientific). One fifth of the cDNA was then used for polymerase chain reaction (PCR) using FastStart Universal SYBR-Green Master Mix (Roche, Basel, Switzerland) on a 7900HT Real-time PCR System (Applied Biosystems, Waltham, MA, USA). GAPDH was used as an endogenous control. The thermal cycling conditions comprised $94^{\circ} \mathrm{C}$ for $30 \mathrm{sec}, 60^{\circ} \mathrm{C}$ for $1 \mathrm{~min}, 72^{\circ} \mathrm{C}$ for $1 \mathrm{~min}$, and final elongation at $72^{\circ} \mathrm{C}$ for $10 \mathrm{~min}$ amplifying for 36 cycles. The primer nucleotide sequences for PCR were designed as follows: GAPDH, forward, 5'-GACGGCCGCATCTTCTTGA-3' and reverse, 5'-CACACCGACCTTCACCATTTT-3'; nuclear factor of activated T-cells 1 (NFATc1) forward, 5'-ACCACCTTTCCGCAA CCA-3' and reverse, 5'-GGTACTGGCTTCTCTTCCGTTTC-3'; TRAP forward, 5'-CTGCTGGGCCTACAAATCATA-3' and reverse, 5'-GGGAGTCCTCAGATCCATAGT-3'. The 2- ${ }^{-\Delta \Delta \mathrm{Cq}}$ method was used for the analysis of the data (20).

Statistical analysis. Data are expressed as the means \pm SEM. Differential levels (apart from the mean density of RANKL expression) were investigated using the Kruskal-Wallis test followed by the Dunn's Multiple Range test for post hoc comparisons. The mean density of RANKL expression levels 
A

DCs

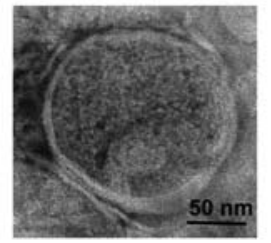

B

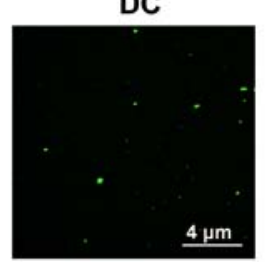

C

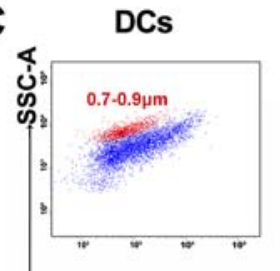

RCs

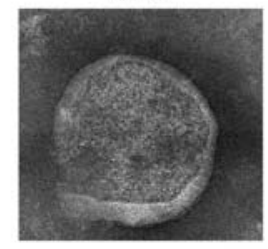

RC

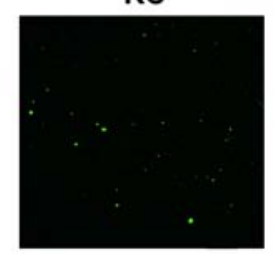

RCs

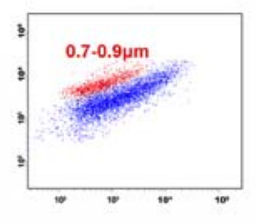

OKCs

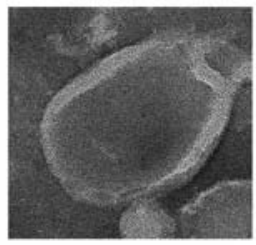

OKC

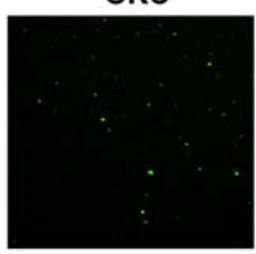

OKCs

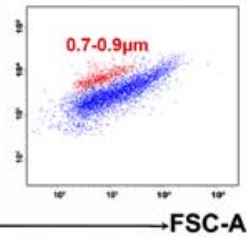

D DCs

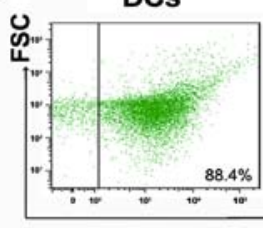
RCs
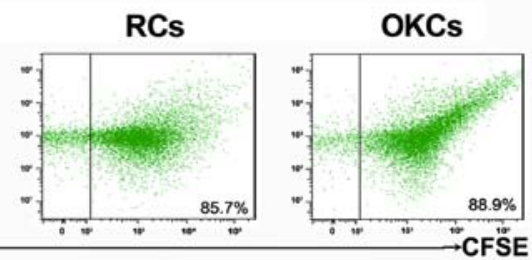

Figure 1. Identification of cyst fluid microparticles (CFMPs) derived from patients with dentigerous cysts (DCs), radicular cysts (RCs) and odontogenic keratocysts (OKCs). (A) Transmission electron microscopy (TEM) images of CFMPs purified from DCs, RCs and OKCs. (B) Fluorescence images showing the successful labeling of CFMPs by the fluorescent dye, carboxyfluorescein succinimidyl ester (CFSE). (C) The size distribution of CFMPs derived from DCs, RCs and OKCs was characterized by flow cytometry based on Nile Red fluorescent particles (red) with known diameters ranging from 0.7-0.9 $\mu \mathrm{m}$. (D) Flow cytometry images showing that CFMPs derived from patients with DCs, RCs and OKCs could be largely stained with CFSE.

in the patient samples were analyzed by the Student's t-test. Spearman's rank correlation analysis was used to investigate the correlation between CFMP levels and RANKL expression in the samples collected from the same patients. For the comparisons, a value of $\mathrm{P}<0.05$ was considered to indicate a statistically significant difference. Statistical analysis was performed using GraphPad Prism 6.0 software.

\section{Results}

Clinical characteristics of the patients with odontogenic cystic lesions. Thirteen patients with DCs ( 7 male, 6 female), 20 patients with RCs (12 male, 8 female) and 26 patients with OKCs (11 male, 15 female) were enrolled in this study (Tables I-III). The average age of the patients with DCs, RCs and OKCs was $36 \pm 12,39 \pm 20$ and $33 \pm 15$ years old, respectively. The average diameters of the DCs, RCs and OKCs were $2.44 \pm 1.38,2,73 \pm 1.80$ and $3.66 \pm 1.53 \mathrm{~cm}$, respectively. No significant differences in age, sex and diameter distribution were found in this study.

Characterization and quantification of CFMPs. CFMPs from patients with DCs, RCs and OKCs were purified by differential centrifugation. To directly visualize the CFMPs, TEM and CFSE fluorescence labeling were performed. As shown in Fig. 1A, the CFMPs were 100-1,000 nm membrane-bounded vesicles with a round or elliptical structure. As shown in Fig. 1B, the CFMPs were successfully stained with CFSE, indicating their membrane structures. Using the Nile Red fluorescent particles with known diameters (0.7-0.9 $\mu \mathrm{m})$, the diameters of the CFMPs were determined ranging from 100 to $1,000 \mathrm{~nm}$ (Fig. 1C). Moreover, the results of flow cytometric analysis demonstrated that the purified CFMPs were successfully detected by CFSE (Fig. 1D). The above-mentioned results suggest that the purified CFMPs are in line with the basic characteristics of MPs.

Cellular origin of MPs in the cyst fluid of DCs, RCs and OKCs. By the addition of one or more CD-specific monoclonal antibodies, we determined the cellular origin of the MPs present in the cyst fluid. Platelet-derived MPs $\left(\mathrm{CD} 31^{+} / \mathrm{CD} 41^{+}\right)$, endothelium-derived MPs (CD144 $\left.{ }^{+} / \mathrm{Annexin}^{+}\right)$, erythro-

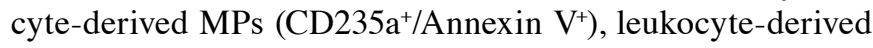
MPs $\left(\mathrm{CD}^{2} 5^{+} / \mathrm{Annexin} \mathrm{V}^{+}\right)$and epithelium-derived MPs $\left(\mathrm{EpCAM}^{+} / \mathrm{Annexin} \mathrm{V}^{+}\right)$were identified in the cyst fluid of DCs, RCs and OKCs. On the other hand, erythrocyte- and 

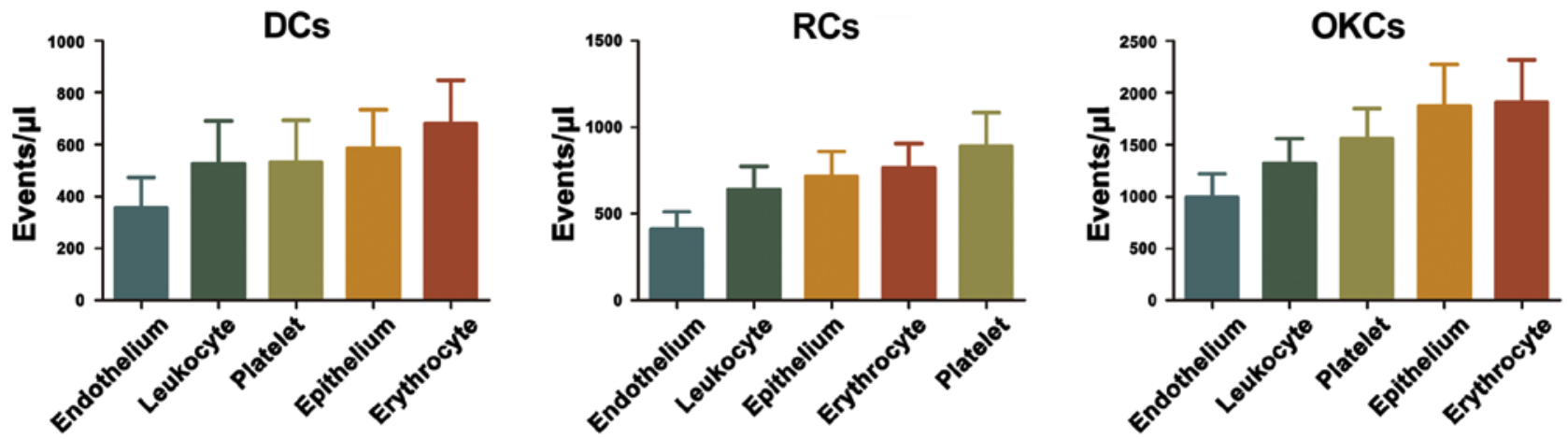

Figure 2. Cellular origin of cyst fluid microparticles (CFMPs). To determine the cellular origin, antibodies were used directly against $\mathrm{CD} 31^{+} / \mathrm{CD} 41^{+}$(platelets), CD235a ${ }^{+} /$Annexin $\mathrm{V}^{+}$(erythrocyte), CD144 ${ }^{+} / \mathrm{Ann}$ exin $\mathrm{V}^{+}$(endothelium), $\mathrm{CD}^{+} 5^{+} /$Annexin $\mathrm{V}^{+}$(leukocyte) and EpCAM ${ }^{+} / \mathrm{Annexin}^{+}$(epithelium). Data are expressed and sorted by the number of microparticles per microlitre.
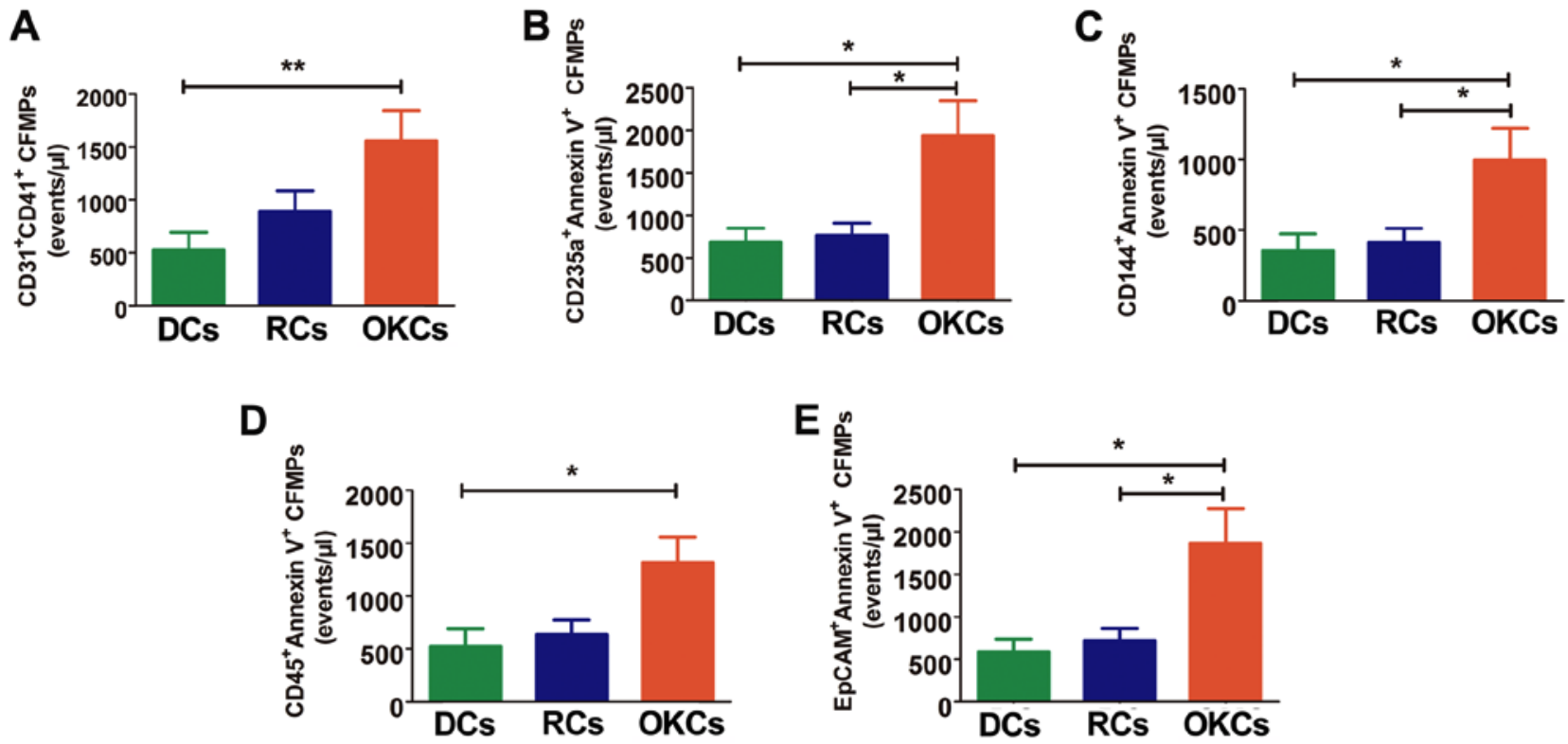

Figure 3. Quantification of the subtypes of cyst fluid microparticles (CFMPs). (A-E) Quantitative analysis of the levels of $\mathrm{CD}^{+} 1^{+} / \mathrm{CD} 41^{+}$(platelets) $\mathrm{CFMPs}$,

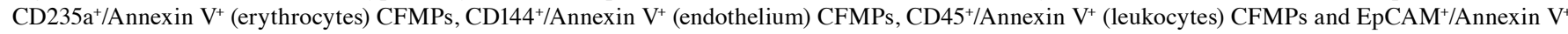
(epithelium) CFMPs in patients with dentigerous cysts (DCs), radicular cysts (RCs) and odontogenic keratocysts (OKCs). Data are expressed as the means $\pm \mathrm{SEM} .{ }^{*} \mathrm{P}<0.05$ vs. the control group.

epithelium-derived MPs were the leading CFMPs from the OKCs and DCs, whereas platelet- and erythrocyte-derived MPs were the top two CFMP subsets from the RCs (Fig. 2). These above-mentioned results showed that these CFMPs shared similar cell origins.

Subtypes of CFMPs are significantly increased in patients with $O K C s$. The results revealed that the levels of leukocyte- and platelet-derived MPs (CD45 $/$ Annexin $\mathrm{V}^{+}$and CD $31^{+} / \mathrm{CD} 41^{+}$, respectively) were significantly increased in the OKCs when compared with the $\mathrm{DCs}(\mathrm{P}<0.05, \mathrm{P}<0.01$ respectively), whereas no significant differences were observed between the DCs and RCs $(\mathrm{P}>0.05)$, and between the OKCs and RCs $(\mathrm{P}>0.05)$ (Fig. 3A and D). Significantly higher levels of erythrocyte-, endothelium- and epithelium-derived CFMPs were also observed in the OKCs when compared with the DCs and RCs $(\mathrm{P}<0.05)$, whereas no significant differences were found between the DCs and RCs (P>0.05) (Fig. 3B, C and E, and Table IV).
The level of CFMPs is increased in patients with OKCs. By applying flow-count beads, the concentrations of CFMPs were determined by flow cytometry (Fig. 4A). The results revealed that the concentration of total CFMPs in the OKCs $(6,751 \pm 5,800$ events $/ \mu 1)$ was significantly higher than that in the RCs $(3,477 \pm 3,346$ events $/ \mu 1 ; \mathrm{P}<0.05)$ and $\mathrm{DCs}$ $(2,755 \pm 2,471$ events $/ \mu \mathrm{l} ; \mathrm{P}<0.05)$ (Fig. $4 \mathrm{~B}$ and Table IV). However, no significant difference was observed in the concentration of CFMPs between the DCs and RCs (P>0.05) (Fig. 4B). The results suggest that the level of CFMPs is elevated in OKCs, which may be associated with the development of the lesions.

CFMPs may promote osteoclastogenesis by upregulating the expression of RANKL in epithelial cells of OKCs. To investigate the potential influence of CFMPs on the osteoclastic activity of odontogenic lesions, we detected the expression level of RANKL in the OKC samples and examined its correlation with the level of CFMPs. According to the mean concentration 
Table IV. Cyst fluid microparticle levels in patients with dentigerous cysts (DCs), radicular cysts (RCs) and odontogenic keratocysts (OKCs).

\begin{tabular}{|c|c|c|c|c|c|}
\hline Variables & OKCs & DCs & $\begin{array}{c}\mathrm{P}<0.05 \\
\mathrm{OKCs} / \mathrm{DCs}\end{array}$ & RCs & $\begin{array}{c}\mathrm{P}<0.05 \\
\mathrm{OKCs} / \mathrm{RCs}\end{array}$ \\
\hline $\mathrm{n}$ & 26 & 13 & ND & 20 & ND \\
\hline $\mathrm{Age}^{\mathrm{a}}$ (years) & $33 \pm 15$ & $36 \pm 12$ & ND & $39 \pm 20$ & ND \\
\hline Sex (male/female) & $11 / 15$ & $7 / 6$ & ND & $12 / 8$ & ND \\
\hline Total MPs ${ }^{\mathrm{b}}$ & $6,751 \pm 5,800$ & $2,755 \pm 2,471$ & $\mathrm{Y}$ & $3,477 \pm 3,346$ & $\mathrm{Y}$ \\
\hline $\mathrm{CD} 31^{+} / \mathrm{CD} 41^{+} \mathrm{MPs}^{\mathrm{b}}$ & $1,559 \pm 1,435$ & $533 \pm 582$ & $\mathrm{Y}$ & $891 \pm 870$ & $\mathrm{~N}$ \\
\hline $\mathrm{CD} 235 \mathrm{a}^{+} \mathrm{MPs}^{\mathrm{b}}$ & $3,212 \pm 3,675$ & $1,464 \pm 1,201$ & $\mathrm{Y}$ & $2,523 \pm 2,863$ & $\mathrm{Y}$ \\
\hline $\mathrm{CD}^{2} 5^{+} \mathrm{MPs}^{\mathrm{b}}$ & $1,319 \pm 1,214$ & $527 \pm 591$ & $\mathrm{Y}$ & $639 \pm 601$ & $\mathrm{~N}$ \\
\hline $\mathrm{CD}_{144^{+}} \mathrm{MPs}^{\mathrm{b}}$ & $996 \pm 1,144$ & $357 \pm 417$ & $\mathrm{Y}$ & $411 \pm 449$ & $\mathrm{Y}$ \\
\hline $\mathrm{EpCAM}^{+} \mathrm{MPs}^{\mathrm{b}}$ & $1,867 \pm 2,072$ & $586 \pm 534$ & Y & $719 \pm 634$ & Y \\
\hline
\end{tabular}

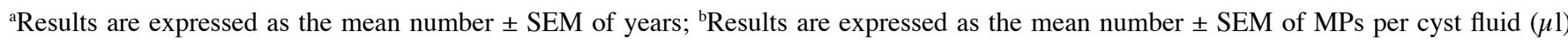
(range). The concentration of MP was determined by flow cytometry analysis using calibrator Flow-Count beads as described in the Materials and methods. ND, not determined; $\mathrm{Y}$, Yes $(\mathrm{P}<0.05)$; N, No $(\mathrm{P}>0.05)$; MPs, microparticles.

A

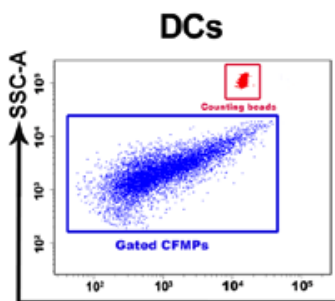

RCs

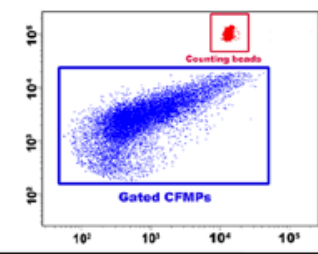

OKCs

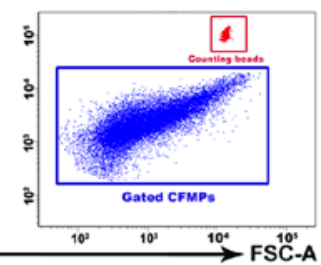

B
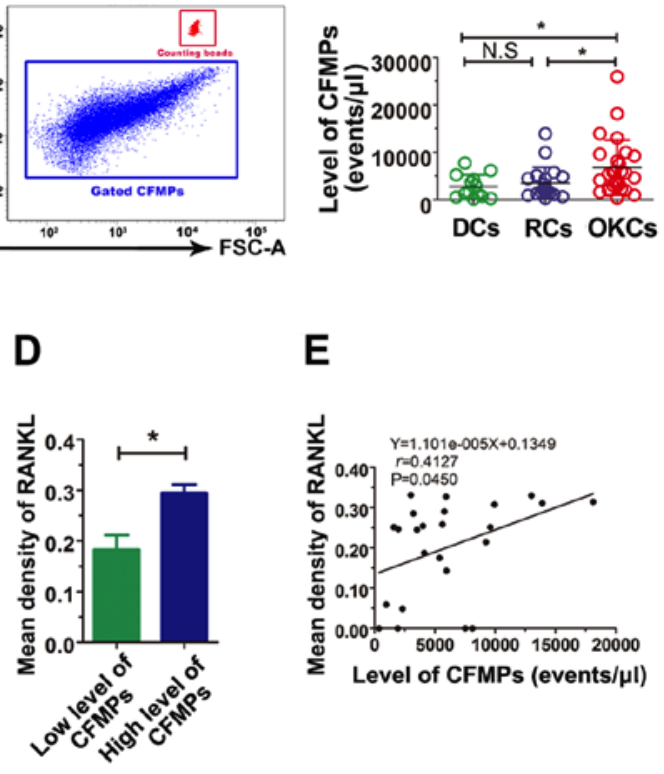

E

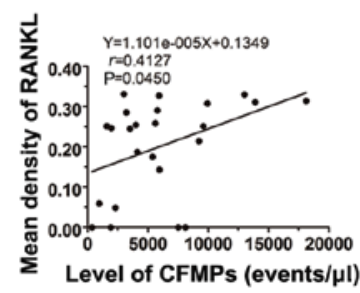

Figure 4. Quantification of the level of cyst fluid microparticles (CFMPs) and the correlation between the level of CFMPs and the clinical characteristics of patients with dentigerous cysts (DCs), radicular cysts (RCs) and odontogenic keratocysts (OKCs). (A) Representative flow cytometry dot-plots showing gated CFMPs (green dots) and the fluorescent beads for counting (red dots). (B) Quantitative analysis of the level of CFMPs derived from patients with DCs, RCs and OKCs. (C) Representative immunohistochemical staining of receptor activator for nuclear factor- $\kappa \mathrm{B}$ ligand (RANKL) in OKCs with different level of CFMPs. (D) Quantitative analysis of staining intensity of RANKL in OKCs with different levels of CFMPs. (E) Spearman's rank correlation test was performed to determine the correlation between the level of CFMPs and the mean density of RANKL in OKCs. The data are expressed as the means \pm SEM. ${ }^{*} \mathrm{P}<0.05$ vs. the control group; N.S, not significant.

of CFMPs, the patients with OKCs were divided into 2 groups, with a high or low level of CFMPs. As shown in Fig. 4C and D, the mean density of RANKL was markedly increased in the patients with a high level CFMPs compared with those with a low level CFMPs $(\mathrm{P}=0.0261)$. The Spearman's rank correlation test revealed that the level of total CFMPs positively correlated with the RANKL expression level in the samples from patients with $\mathrm{OKCs}(\mathrm{r}=0.4127, \mathrm{P}=0.0450)$ (Fig. 4E). As shown in Fig. 5A, the CFMPs were detected in the HIOECs at $2 \mathrm{~h}$. In addition, the results from DNA electrophoresis revealed that the CFMPs from the OKCs expressed RANKL mRNA (Fig. 5B). In addition, the HIOECs exhibited a higher protein expression level of RANKL when compared with the control group (Fig. 5C). Previous studies have demonstrated that RANKL is expressed in the components of odontogenic lesions and contributes to the bone destruction of OKCs $(21,22)$. 

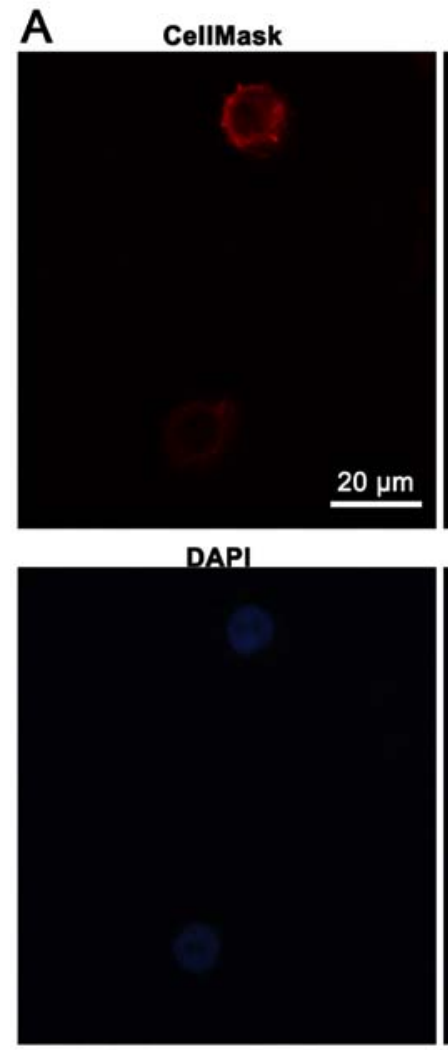
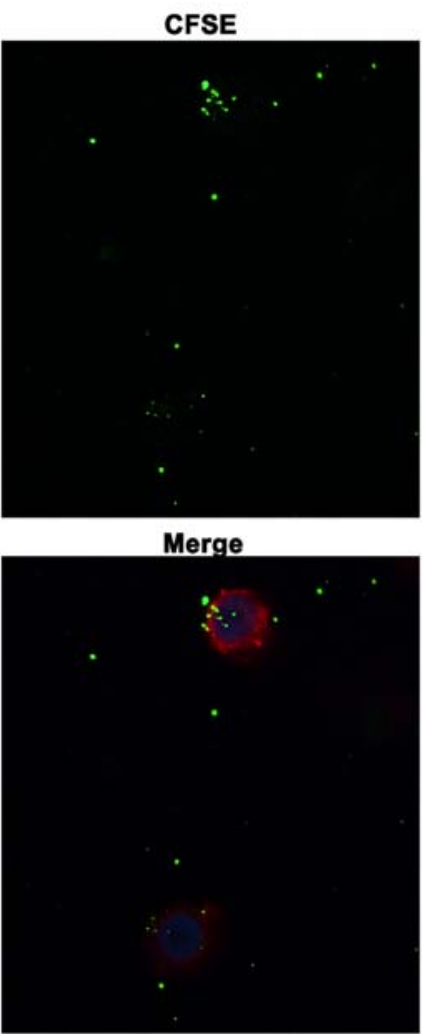

\section{B}

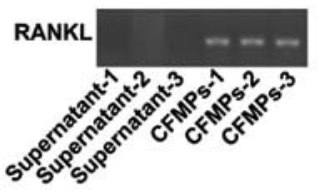

\section{C}

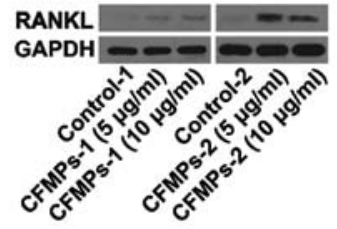

Figure 5. Effect of cyst fluid microparticles (CFMPs) isolated from two patients with odontogenic keratocysts (OKCs) on human immortalized oral epithelial cells (HIOECs). (A) Uptake assays for CFSE-labeled CFMPs (green) were performed by using HIOECs (red) as the recipient cells. (B) DNA electrophoresis indicated that CFMPs from OKCs expressed receptor activator for nuclear factor- $\mathrm{kB}$ ligand (RANKL) mRNA. (C) RANKL expression was examined by western blot analysis in CFMPs co-cultured with HIOECs. The CFMPs were selected from two patients with OKCs.
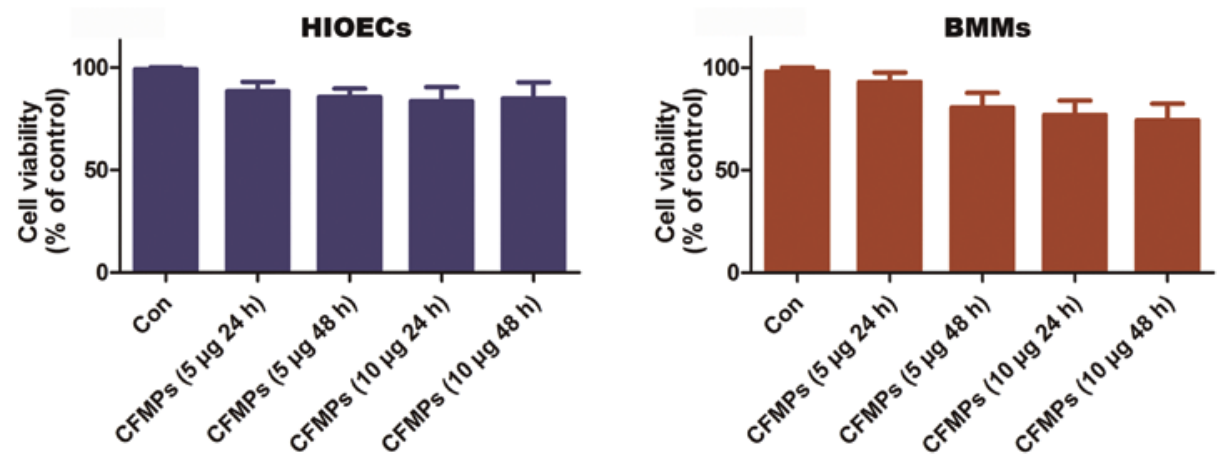

Figure 6. Cells were treated with or without CFMPs (5 or $10 \mu \mathrm{g} / \mathrm{ml}$ ) in a 96 -well plate for 24 or $48 \mathrm{~h}$. Cell viability was measured by MTT assay. Results were expressed as the percentage (\%) viability of cells compared to the control (means \pm SEM, $n=5$ ). P-values $<0.05$ were considered to indicate statistically significant differences. No significant differences were observed in this analysis. The results shown here as a representative of 3 independent experiments. HIOECs, human immortalized oral epithelial cells; BMMs, bone marrow-derived macrophages.

Our results thus suggested that the increased levels of CFMPs were closely associated with the expression of RANKL in the epithelium of OKCs, indicating that the CFMPs may be related to the osteoclastogenesis of OKCs.

CFMPs have no effects on the proliferative ability of HIOECS and BMMs. The HIOECs and BMMs were co-cultured with or without various concentrations of CFMPs (5 or $10 \mu \mathrm{g} / \mathrm{ml})$ for 24 or $48 \mathrm{~h}$. Cell viability was determined by MTT assay. The results showed that the CFMPs (5 and $10 \mu \mathrm{g} / \mathrm{ml}$ ) had no effect on the viability of the HIOECs and BMMs when compared to the control group (Fig. 6).
CFMPs isolated from OKCs promote the osteoclastogenesis of BMMs. To further determine the biological effects of CFMPs, a series of in vitro experiments using mouse BMMs were carried out. The results of western blot analysis and flow cytometry confirmed that the CFMPs from the OKCs exhibited a higher expression of RANKL (Fig. 7). Therefore, we examined whether the CFMPs could promote the osteoclastogenesis of BMMs. As shown in Fig. 8A, following incubation with CFSE-labeled CFMPs for $2 \mathrm{~h}$, green fluorescence dots were detected in the CellMask-stained BMMs, indicating that the CFMPs were effectively taken up by the BMMs. In addition, as shown in Fig. 8B, the osteoclasts, characterized 

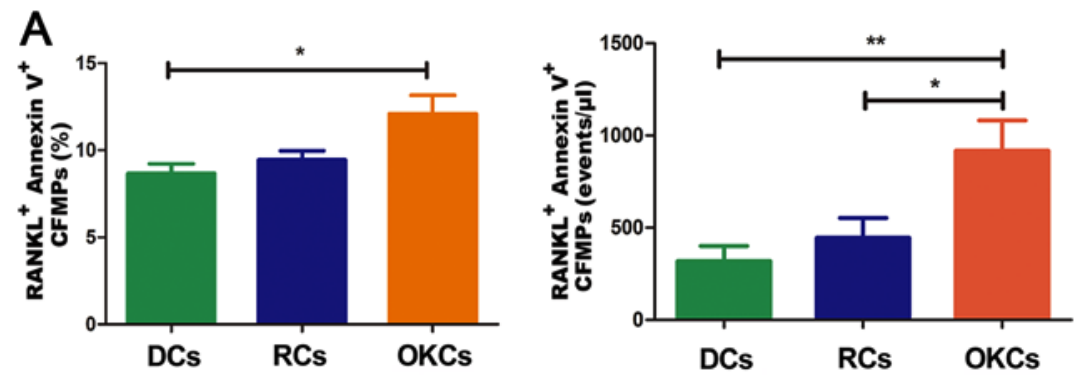

B

EрСАM

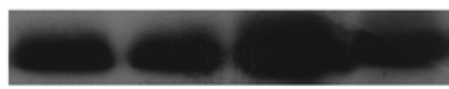

RANKL

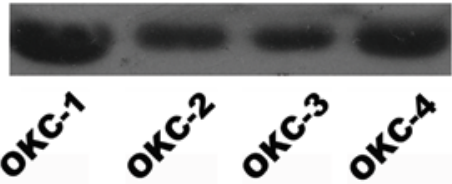

Figure 7. Receptor activator for nuclear factor- $\mathrm{KB}$ ligand (RANKL) expression in cyst fluid microparticles (CFMPs) of jaw cysts. (A) Statistic analysis showing the RANKL $\mathrm{R}^{+}$Annexin $\mathrm{V}^{+}$microparticles in patients with dentigerous cysts (DCs), radicular cysts (RCs) and odontogenic keratocysts (OKCs). (B) The protein level of RANKL was examined by western blot analysis of the CFMPs from 4 patients with OKCs.

A
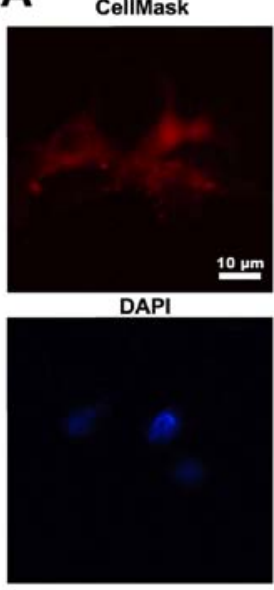

C

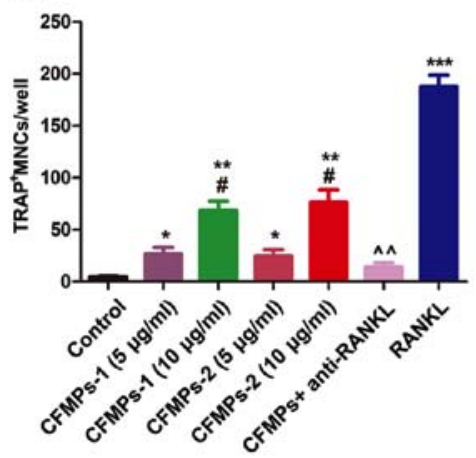

CFSE
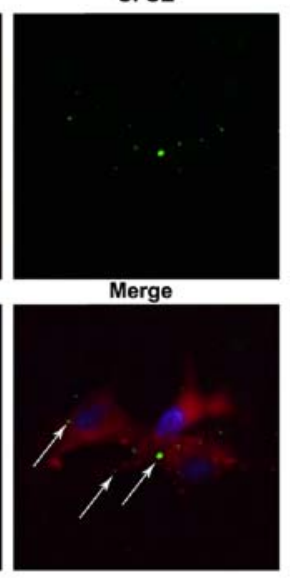

B

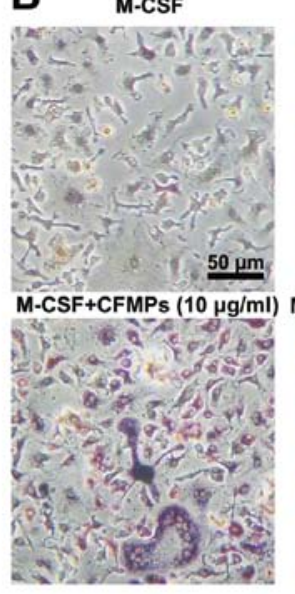

M-CSF+CFMPs $(5 \mu \mathrm{g} / \mathrm{ml})$

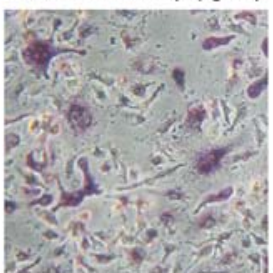

M-CSF+CFMPs+anti-RANKL

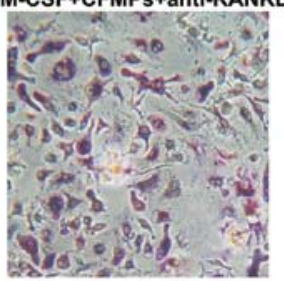

M-CSF+RANKL

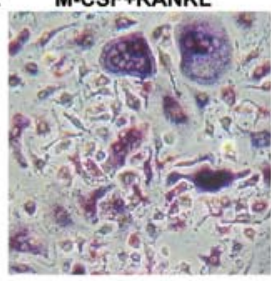

D

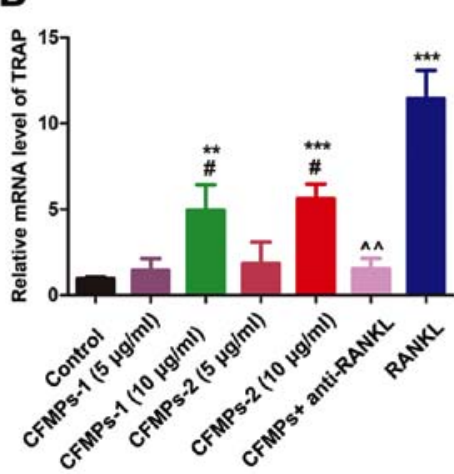

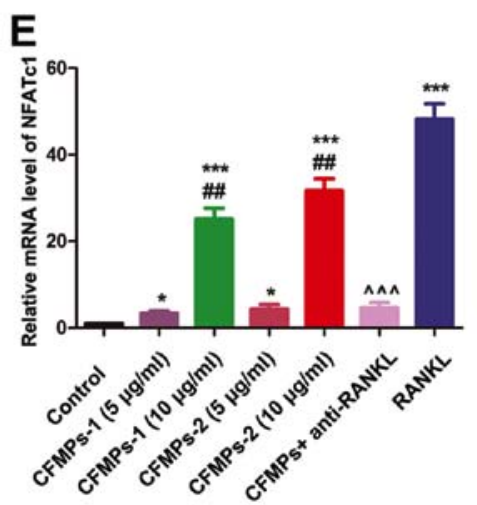

Figure 8. Effect of cyst fluid microparticles (CFMPs) isolated from 2 patients with odontogenic keratocysts (OKCs) on the osteoclastogenesis of bone marrow-derived macrophages (BMMs). (A) Uptake assays for CFSE-labeled CFMPs (green) were performed by using BMMs (labeled with CellMask) as the recipient cells. (B) Tartaric-resistant acid phosphatase (TRAP) staining showed that multinucleated osteoclasts were observed in the presence of macrophage colony-stimulating factor (M-CSF) $(20 \mathrm{ng} / \mathrm{ml})$ and CFMPs (5 or $10 \mu \mathrm{g} / \mathrm{ml})$. The anti-receptor activator for nuclear factor- $\mathrm{kB}$ ligand (RANKL) monoclonal antibody was used to neutralize the RANKL protein. The soluble RANKL (sRANKL; $50 \mathrm{ng} / \mathrm{ml}$ )-treated group was selected as a positive control. (C) Quantification analysis of the results from TRAP staining. (D and E) The mRNA levels of the nuclear factor of activated T-cells 1 (NFATc1) and TRAP were evaluated by RT-qPCR in BMMs co-cultured with various concentrations of CFMPs. The M-CSF (20 ng/ml)- and sRANKL (50 ng/ml)-treated group was used as a positive control. Data are shown as the means \pm SEM. ${ }^{*} \mathrm{P}<0.05,{ }^{* *} \mathrm{P}<0.01$ and ${ }^{* * * *} \mathrm{P}<0.001$ vs. the control group. ${ }^{\#} \mathrm{P}<0.05$ and ${ }^{\# \#} \mathrm{P}<0.01$ vs. the $\mathrm{CFMP}$ $(5 \mu \mathrm{g} / \mathrm{ml})$-treated group from the same patient. ${ }^{\wedge} \mathrm{P}<0.05$ and ${ }^{\wedge \wedge} \mathrm{P}<0.01 \mathrm{vs}$. the CFMP $(10 \mu \mathrm{g} / \mathrm{ml})$-treated group from the same patient. 
by multinuclear giant cells and TRAP positive cells, were detected in the M-CSF- and CFMP-treated group or the M-CSF- and sRANKL-treated group. The quantification of TRAP staining demonstrated that the CFMPs derived from OKCs significantly enhanced the osteoclastogenesis of the BMMs in a concentration-dependent manner (Fig. 8C). Additionally, the results of RT-qPCR suggested that the CFMPs significantly promoted the mRNA expression levels of representative markers for osteoclast differentiation, TRAP and NFATc1 (Fig. 8D and E). Moreover, the anti-RANKL monoclonal antibody $(1,000 \mathrm{ng} / \mathrm{ml})$ - and CFMP $(10 \mu \mathrm{g} / \mathrm{ml})$-treated group exhibited lower numbers of TRAP-positive cells, and lower mRNA levels of TRAP and NFATc1. This indicated that CFMPs may promote the osteoclastogenesis of BMMs through RANKL carried by CFMPs.

\section{Discussion}

To the best of our knowledge, this study demonstrates for the first time, the presence of MPs shed from platelets, endothelial cells, leukocytes, erythrocytes and epithelial cells into the cyst fluids of patients with DCs, RCs and OKCs, and demonstrates the elevation of CFMPs in patients with OKCs. The levels of CFMPs are closely associated with the diameters of lesions and RANKL expression levels in OKC tissues. In addition, we demonstrate that CFMPs isolated from patients with $\mathrm{OKCs}$ stimulate BMM differentiation and lead to the formation of osteoclasts, suggesting that CFMPs may contribute to the osteoclastogenesis of OKCs.

Cyst fluid is a crucial component of the microenvironment of odontogenic cysts. The levels of proteins and/or cytokines within the cyst fluid of OKCs have been shown to be quite different from those in DCs or RCs. For instance, it has been reported that the protein level of lactoferrin is significantly higher in OKCs compared with other cysts, due to the impermeability nature of the cyst wall of OKCs (23-25). Epithelial cells are more frequently present in the aspirates of OKCs when compared with those of DCs and RCs (26). Moreover, there is evidence to indicate that the cyst fluid level of interleukin (IL)-1 $\alpha$ in OKCs is significantly higher than that in DC or RC fluids, associated with a higher expression level of matrix metalloproteinase-9 (MMP9) in the OKC epithelium $(27,28)$. In addition, the higher level of transforming growth factor- $\beta$ (TGF- $\beta$ ) within the fluids plays a significant role in inducing RANKL expression in the stroma, which is essential for the osteoclastogenesis of OKCs (29). These data suggest that different components within the cyst fluids may contribute to the development of OKCs. A number of studies have demonstrated that MPs, which are membrane vesicles produced upon cell apoptosis or activation (7), are present in several human body fluids, such as plasma, saliva and synovial fluid (30-32). These MPs naturally inherit membrane lipids, surface antigens, cytoplasmic proteins as well as nucleic acids from their donor cells, which could be transferred into the target cell, and then affect various cell functions (33). However, whether MPs are present in the cyst fluid of OKCs and their role in the development of OKCs remains unknown.

During the release of MPs, the asymmetric distribution of phospholipids in the two leaflets of the plasma membrane is lost, leading to phospholipid exposure (34). Typically,
MPs expose the anionic phospholipid PS on their membrane surface, enabling their detection by Annexin V staining (14). The cyst fluid of OKCs usually presents as a semi-solid form, and is characterized by a large amount of keratins (35). Due to the similar size distributions, it is a great challenge to distinguish MPs from protein fragments within the body fluids, which otherwise results in a significant amount of the background noise for the quantification of MPs (36). Therefore, in this study, we defined CFMPs using positive staining for Annexin V in order to distinguish MPs from cell debris or precipitates according to a previous protocol (36). In addition, as MPs carry surface membrane antigens from their donor cells, we tested the various cellular origins of MPs using Annexin V and specific markers, such as CD144 (endotheliocyte marker), CD235a (erythrocyte marker), EpCAM (epithelium marker) or CD45 (leukocyte marker). In this study, at least to the best of our knowledge, we demonstrate for the first time that MPs are present in the cyst fluid of OKCs, and have a wide origin of different cells including blood cells, epithelial cells and inflammatory cells. Moreover, the results demonstrated that all the subtypes of CFMPs were significantly elevated in OKCs when compared with DCs and RCs. The majority of previous studies have shown that the elevated level of body fluid MPs in patients bearing tumors may be caused by the neoplastic characteristics, such as highly cell activation, apoptosis abnormalities or chronic inflammation $(37,38)$. Although the precise mechanisms behind the elevated level of CFMPs remain to be elucidated, there are two possible reasons for the higher level of CFMPs within OKCs. First, the aberrant proliferation and apoptotic characteristics of the OKC epithelium lead to the increased release of MPs, which is similar to other tumor lesions $(12,39)$. This could explain the elevation of EpCAM ${ }^{+}$MPs in OKCs compared with DCs and RCs. However, it cannot fully account for the higher level of MPs derived from the blood or inflammatory cells, such as CD $235 \mathrm{a}^{+}, \mathrm{CD} 31^{+} / \mathrm{CD} 41^{+}$and CD $45^{+}$CFMPs. Second, as previous studies have confirmed that the relative impermeability of the capsule wall of OKCs could lead to specific accumulations of proteins (23-25), it is possible that MPs, which contain larger amounts of proteins, are also accumulated in the cyst cavity due to the impermeability of the capsule wall of OKCs. This may better explain the entire elevations of various MP types in OKC cyst fluids.

For all the odontogenic cysts or tumors, bone resorption is one of the critical events (40). It is a complex process initiated by the proliferation of immature osteoclasts. The interaction between RANKL and RANK promotes the osteoclast differentiation by activating the intracellular signaling (41). In another odontogenic tumor named ameloblastoma, it has been suggested that the co-culture of ameloblastoma cells and rabbit bone marrow cells induces osteoclastogenesis via the RANK/RANK/OPG signaling pathway (22). MPs carry RANKL protein and can be internalized and deliver their cargo into the cytosol of recipient cells, thereby activating or inhibiting specific signaling pathways (42). Previous studies have demonstrated that cytokines can induce the mRNA level of RANKL and therefore boost the protein level of RANKL in oral epithelial cells (43). In exploring the roles of CFMPs, we found that the level of CFMPs was closely related with RANKL expression within the epithelium of OKCs. Furthermore, 
CFMPs can promote the expression of RANKL in HIOECs which may be closely associated with the mRNA of RANKL contained in CFMPs. Our results indicated that CFMPs may be representative of the tissues, and may contribute to the bone resorption of OKCs. However, the function of CFMPs in vitro remains to be determined.

Of note, we found that BMMs could easily uptake CFMPs which contained RANKL $\mathrm{mRNA}$ and protein at $2 \mathrm{~h}$, indicating that CFMPs may exert biological effects on the recipient cells. To investigate the biological functions of CFMPs, CFMPs were added to the BMMs and the levels of osteoclastogenesis-related genes, such as TRAP and NFATc1 were found to be significantly elevated in the BMMs co-cultured with CFMPs. More importantly, we found that BMMs could successfully differentiate into osteoclasts in the presence of M-CSF and CFMPs, which may be a novel mechanism of osteoclastogenesis in OKCs. The osteoclasts absorb the adjacent bone to acquire the space of the cavity for the growth of the lesion. This may also imply the close association between the CFMP level and RANKL expression in the tissue. Taken together, our study demonstrates that the level of CFMPs may be an important indicator of the progression of OKCs.

In conclusion, the present study demonstrated that the level of CFMPs was significantly elevated in OKCs and was closely associated with cyst diameters. In vitro experiments revealed that CFMPs could be internalized by BMMs, leading to increased mRNA expression levels of NFATc1 and TRAP in the BMMs. Further studies are warranted in order to elucidate the precise mechanisms underlying the alternations in CFMP profiles and the functional significance of CFMPs.

\section{Acknowledgements}

The authors would like to thank the technician, Juan Min, from the Wuhan Institute of Virology, Chinese Academy of Sciences for supporting our flow cytometric analysis. The authors would also like to thank Professor San-Gang He from the School of Stomatology, Wuhan University for providing the HIOECs.

\section{Funding}

This study was supported by grants from the National Natural Science Foundation of China to GC (no. 81671816) and YFZ (no. 81570994).

\section{Availability of data and materials}

The datasets used and/or analyzed during the current study are available from the corresponding author on reasonable request.

\section{Authors' contributions}

QWM contributed to the design of the study and wrote the manuscript. QWM, YYZ and JYL collected the clinical samples. QWM, JGR and WQZ performed the flow cytometry analysis and CFMP identification. RFL and BFN performed the cell experiments. YFZ, GC and BL performed the data analysis and revised the manuscript. All authors have read and approved this manuscript.

\section{Ethics approval and consent to participate}

The use of human samples was approved by the Review Board of the Medical Ethics Committee of the Hospital of Stomatology, Wuhan University, Wuhan, China. All patients agreed to participate in the study and signed informed consent forms. The use of animals was approved by the Medical Ethics Committee of Hospital of Stomatology, Wuhan University.

\section{Consent for publication}

Not applicable.

\section{Competing interests}

The authors declare that they have not competing interests.

\section{References}

1. Browne RM: The pathogenesis of odontogenic cysts: A review. J Oral Pathol 4: 31-46, 1975.

2. Kramer IR, Pindborg JJ and Shear M: The WHO histological typing of odontogenic tumours. A commentary on the second edition. Cancer 70: 2988-2994, 1992.

3. Avelar RL, Antunes AA, Carvalho RW, Bezerra PG, Oliveira Neto PJ and Andrade ES: Odontogenic cysts: A clinicopathological study of 507 cases. J Oral Sci 51: 581-586, 2009.

4. Lin HP, Wang YP, Chen HM, Cheng SJ, Sun A and Chiang CP: A clinicopathological study of 338 dentigerous cysts. J Oral Pathol Med 42: 462-467, 2013.

5. Thompson L: World Health Organization classification of tumours: Pathology and genetics of head and neck tumours. Ear Nose Throat J 85: 74, 2006

6. Wright JM and Vered M: Update from the 4th edition of the World Health Organization Classification of head and neck tumours: Odontogenic and maxillofacial bone tumors. Head Neck Pathol 11: 68-77, 2017

7. 7Abels ER and Breakefield XO: Introduction to extracellular vesicles: Biogenesis, RNA cargo selection, content, release and uptake. Cell Mol Neurobiol 36: 301-312, 2016.

8. Ren JG, Man QW, Zhang W, Li C, Xiong XP, Zhu JY, Wang WM, Sun ZJ, Jia J, Zhang WF, et al: Elevated Level of Circulating Platelet-derived Microparticles in Oral Cancer. J Dent Res 95: 87-93, 2016.

9. Ren JG, Zhang W, Liu B, Man QW, Xiong XP, Li C, Zhu JY, Wang WM, Jia J, Sun ZJ, et al: Clinical Significance and Roles in Angiogenesis of Circulating Microparticles in Oral Cancer. J Dent Res 95: 860-867, 2016.

10. Chen CL, Lai YF, Tang P, Chien KY, Yu JS, Tsai CH, Chen HW, Wu CC, Chung T, Hsu CW, et al: Comparative and targeted proteomic analyses of urinary microparticles from bladder cancer and hernia patients. J Proteome Res 11: 5611-5629, 2012.

11. Press JZ, Reyes M, Pitteri SJ, Pennil C, Garcia R, Goff BA, Hanash SM and Swisher EM: Microparticles from ovarian carcinomas are shed into ascites and promote cell migration. Int J Gynecol Cancer 22: 546-552, 2012.

12. Roca E, Lacroix R, Judicone C, Laroumagne S, Robert S, Cointe S, Muller A, Kaspi E, Roll P, Brisson AR, et al: Detection of EpCAM-positive microparticles in pleural fluid: A new approach to mini-invasively identify patients with malignant pleural effusions. Oncotarget 7: 3357-3366, 2016.

13. Tkach $\mathrm{M}$ and Théry $\mathrm{C}$ : Communication by extracellular vesicles: Where we are and where we need to go. Cell 164: 1226-1232, 2016.

14. Mallat Z, Hugel B, Ohan J, Lesèche G, Freyssinet JM and Tedgui A: Shed membrane microparticles with procoagulant potential in human atherosclerotic plaques: A role for apoptosis in plaque thrombogenicity. Circulation 99: 348-353, 1999.

15. Burnier L, Fontana P, Kwak BR and Angelillo-Scherrer A: Cell-derived microparticles in haemostasis and vascular medicine. Thromb Haemost 101: 439-451, 2009.

16. Nieuwland R, Berckmans RJ, McGregor S, Böing AN, Romijn FP, Westendorp RG, Hack CE and Sturk A: Cellular origin and procoagulant properties of microparticles in meningococcal sepsis. Blood 95: 930-935, 2000. 
17. Mendes RA, Carvalho JF and van der Waal I: Characterization and management of the keratocystic odontogenic tumor in relation to its histopathological and biological features. Oral Oncol 46: 219-225, 2010.

18. Zhao JY, Chen G, Gu YP, Cui R, Zhang ZL, Yu ZL, Tang B, Zhao YF and Pang DW: Ultrasmall magnetically engineered $\mathrm{Ag} 2 \mathrm{Se}$ quantum dots for instant efficient labeling and whole-body high-resolution multimodal real-time tracking of cell-derived microvesicles. J Am Chem Soc 138: 1893-1903, 2016.

19. Grisendi G, Finetti E, Manganaro D, Cordova N, Montagnani G, Spano C, Prapa M, Guarneri V, Otsuru S, Horwitz EM, et al: Detection of microparticles from human red blood cells by multiparametric flow cytometry. Blood Transfus 13: 274-280, 2015.

20. Livak KJ and Schmittgen TD: Analysis of relative gene expression data using real-time quantitative PCR and the 2(-Delta Delta C(T)) method. Methods 25: 402-408, 2001.

21. da Silva TA, Batista AC, Mendonça EF, Leles CR, Fukada S and Cunha FQ: Comparative expression of RANK, RANKL, and OPG in keratocystic odontogenic tumors, ameloblastomas, and dentigerous cysts. Oral Surg Oral Med Oral Pathol Oral Radiol Endod 105: 333-341, 2008

22. Qian Y and Huang HZ: The role of RANKL and MMP-9 in the bone resorption caused by ameloblastoma. J Oral Pathol Med 39: $592-598,2010$

23. Douglas CW and Craig GT: Quantitation of lactoferrin in odontogenic cyst fluids. J Clin Pathol 42: 180-183, 1989.

24. Jurisic V, Terzic T, Colic S and Jurisic M: The concentration of TNF-alpha correlate with number of inflammatory cells and degree of vascularization in radicular cysts. Oral Dis 14: 600-605, 2008.

25. Skaug N: Proteins in fluid from non-keratinizing jaw cysts. 4 Concentrations of immunoglobulins ( $\operatorname{IgG}, \operatorname{IgA}$ and $\operatorname{IgM}$ ) and some non-immunoglobulin proteins: Relevance to concepts of cyst wall permeability and clearance of cystic proteins. J Oral Pathol 3: 47-61, 1974.

26. Patidar M, Shetty P, Patidar N, Mittal S, Singh H and Chethna: Biochemical and cytological comparison of keratocystic odontogenic tumours to nonkeratinising odontogenic cysts fluid. J Clin Diagn Res 9: ZC34-ZC38, 2015.

27. Ninomiya T, Kubota Y, Koji T and Shirasuna K: Marsupialization inhibits interleukin-1alpha expression and epithelial cell proliferation in odontogenic keratocysts. J Oral Pathol Med 31: 526-533, 2002

28. Kubota Y, Ninomiya T, Oka S, Takenoshita Y and Shirasuna K: Interleukin-1alpha-dependent regulation of matrix metalloproteinase-9(MMP-9) secretion and activation in the epithelial cells of odontogenic jaw cysts. J Dent Res 79: 1423-1430, 2000.

29. Yamada C, Aikawa T, Okuno E, Miyagawa K, Amano K, Takahata S, Kimata M, Okura M, Iida S and Kogo M: TGF- $\beta$ in jaw tumor fluids induces RANKL expression in stromal fibroblasts. Int J Oncol 49: 499-508, 2016.

30. Berckmans RJ, Sturk A, van Tienen LM, Schaap MC and Nieuwland R: Cell-derived vesicles exposing coagulant tissue factor in saliva. Blood 117: 3172-3180, 2011.
31. Berckmans RJ, Nieuwland R, Tak PP, Böing AN, Romijn FP, Kraan MC, Breedveld FC, Hack CE and Sturk A: Cell-derived microparticles in synovial fluid from inflamed arthritic joints support coagulation exclusively via a factor VII-dependent mechanism. Arthritis Rheum 46: 2857-2866, 2002.

32. Berckmans RJ, Nieuwland R, Böing AN, Romijn FP, Hack CE and Sturk A: Cell-derived microparticles circulate in healthy humans and support low grade thrombin generation. Thromb Haemost 85: 639-646, 2001.

33. van der Pol E, Böing AN, Harrison P, Sturk A and Nieuwland R: Classification, functions, and clinical relevance of extracellular vesicles. Pharmacol Rev 64: 676-705, 2012.

34. Piccin A, Murphy WG and Smith OP: Circulating microparticles: Pathophysiology and clinical implications. Blood Rev 21: 157-171, 2007.

35. Aragaki T, Michi Y, Katsube K, Uzawa N, Okada N, Akashi T, Amagasa T, Yamaguchi A and Sakamoto K: Comprehensive keratin profiling reveals different histopathogenesis of keratocystic odontogenic tumor and orthokeratinized odontogenic cyst. Hum Pathol 41: 1718-1725, 2010.

36. Dey-Hazra E, Hertel B, Kirsch T, Woywodt A, Lovric S, Haller H, Haubitz M and Erdbruegger U: Detection of circulating microparticles by flow cytometry: Influence of centrifugation, filtration of buffer, and freezing. Vasc Health Risk Manag 6: 1125-1133, 2010.

37. Becker A, Thakur BK, Weiss JM, Kim HS, Peinado H and Lyden D: Extracellular vesicles in cancer: Cell-to-cell mediators of metastasis. Cancer Cell 30: 836-848, 2016.

38. Todorova D, Simoncini S, Lacroix R, Sabatier F and DignatGeorge F: Extracellular vesicles in angiogenesis. Circ Res 120: 1658-1673, 2017.

39. Kichi E, Enokiya Y, Muramatsu T, Hashimoto S, Inoue T, Abiko Y and Shimono M: Cell proliferation, apoptosis and apoptosisrelated factors in odontogenic keratocysts and in dentigerous cysts. J Oral Pathol Med 34: 280-286, 2005.

40. Main DM: The enlargement of epithelial jaw cysts. Odontol Revy 21: $29-49,1970$

41. Wada T, Nakashima T, Hiroshi $\mathrm{N}$ and Penninger JM: RANKL-RANK signaling in osteoclastogenesis and bone disease. Trends Mol Med 12: 17-25, 2006.

42. Deng L, Wang Y, Peng Y, Wu Y, Ding Y, Jiang Y, Shen Z and Fu Q: Osteoblast-derived microvesicles: A novel mechanism for communication between osteoblasts and osteoclasts. Bone 79: 37-42, 2015.

43. Fujihara R, Usui M, Yamamoto G, Nishii K, Tsukamoto Y, Okamatsu Y, Sato T, Asou Y, Nakashima K and Yamamoto M: Tumor necrosis factor- $\alpha$ enhances RANKL expression in gingival epithelial cells via protein kinase A signaling. J Periodontal Res 49: 508-517, 2014. 